\title{
Flight data monitoring/tracker system for search and rescue mission
}

\begin{abstract}
Traditionally, Kalman Filter is used for the purpose of mixing several input signals and extracting a more reliable output, which greatly benefits aircraft navigation. This paper considers a fusion of four sensor systems: Global Positioning System (GPS), accelerometer, gyroscope and magnetometer. The resultant device, known as Starfish Main Tracking Unit (MTU), is a Flight Data Monitoring (FDM) / Tracking System equipment that uses General Packet Radio Service (GPRS) / Iridium / ICS (Internet Communications Services), which provides low cost telemetry as well as multiple solutions for global flight following and flight data transfer between aircraft and ground. Users from ground are able to monitor their fleet, configure their systems and also generate various flight reports from a single web-based interface, named the Starfish Fleet Management system. This developed system complements the Black Box by downloading limited aircraft data to the ground, provides real time tracking and assist in Search and Rescue (SAR) mission.
\end{abstract}

Keyword: Aircraft navigation; FDM; Flight tracker; GPS; Kalman filters; Sensor fusion 